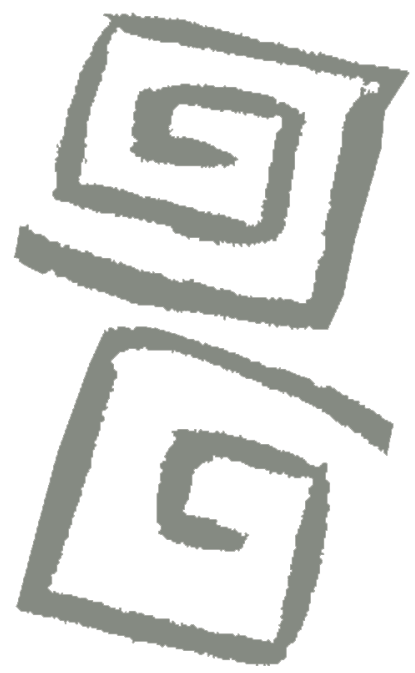

\title{
Formación Paideia en atención primaria: análisis de la democracia institucional y las relaciones de poder en las prácticas laborales en salud
}

\author{
Paideia Education in primary care: an analysis \\ of institutional democracy and power relations \\ in health sector work practices
}

Mônica Martins de Oliveira Viana', Lilian Soares Vidal Terra²

\begin{abstract}
${ }^{1}$ Autora de correspondencia. Doctora en Salud Colectiva. Investigadora, Instituto de Saúde, Secretaria de Estado da Saúde, São Paulo; Brasil. $\triangle$ (iD)

${ }^{2}$ Magíster en Salud Colectiva, Políticas y Gestión en Salud; Departamento de Saúde Coletiva, Universidade Estadual de Campinas, Campinas, São Paulo, Brasil. $\Delta$ iD
\end{abstract}

RESUMEN Este artículo parte de dos iniciativas pedagógicas de "formación Paideia" con el propósito de analizar sus contribuciones en la producción de relaciones democráticas y en la coproducción del cuidado en atención primaria de la salud. La primera iniciativa realizó una capacitación en apoyo matricial (2015-2016) que reunió a 94 especialistas y, la segunda, una capacitación en salud familiar (2018-2020), que reunió a 149 profesionales. Los 243 profesionales de la salud participantes provenían de cinco municipios brasileños. Los resultados obtenidos a través de cuestionarios y diarios de campo elaborados durante los cursos señalan que la formación Paideia motiva a los profesionales a incorporar una clínica ampliada y el apoyo matricial en atención primaria de la salud, y propicia compartir el cuidado y la co-construcción de la autonomía junto a los usuarios. Sin embargo, aún son frecuentes las prácticas autoritarias de la gestión hacia las y los profesionales, entre profesionales, y desde las y los profesionales hacia las personas usuarias, de modo que es necesario invertir, más allá de la formación, en una mayor democratización de los servicios.

PALABRAS CLAVES Atención Primaria de Salud; Capacitación de Recursos Humanos en Salud; Sistema Único de Salud; Relaciones Profesional-Paciente; Brasil.

\begin{abstract}
This article looks at two pedagogical initiatives based on "Paideia Education" in order to analyze their contributions to the production of democratic relations and the co-production of care in primary health care. The first of these initiatives was a seminar on matrix support (2015-2016) that trained 94 specialists, and the second was a seminar on family care (2018-2020) that trained 149 professionals. The 243 participating health professionals came from five different municipalities in Brazil. The results obtained through questionnaires and field diaries kept during the seminars indicate that "Paideia Education" has the ability to motivate professionals to incorporate notions such as the expanded clinic and matrix support into primary health care, and fosters the sharing of care and the co-construction of autonomy with users. However, authoritarian management practices are still frequently directed towards professionals, practiced among professionals themselves, and directed towards patients, and therefore it is necessary to invest in a greater democratization of services beyond training.
\end{abstract}

KEY WORDS Primary Health Care; Health Human Resource Training; Unified Health System; Professional-Patient Relations; Brazil. 


\section{INTRODUCCIÓN}

La atención primaria de la salud (APS) representa un punto fundamental para la estructuración de las redes de atención en el contexto de los sistemas universales de salud, como el Sistema Único de Salud (SUS) en Brasil ${ }^{(1)}$. Sin embargo, para que la APS pueda consagrarse en su función de promover la salud integral, su funcionamiento debe fundarse en los supuestos definidos por Giovanella y Mendonça ${ }^{(2)}$ como APS integral, teniendo como principales atributos: la capilaridad en el territorio para ser efectiva como primer contacto en el acceso del usuario, longitudinalidad, integralidad, coordinación del cuidado, orientación a la comunidad, centralidad en la familia y competencia cultural. Según dichas autoras, una APS integral reconoce su papel para enfrentar los determinantes sociales de la salud y para ser parte de la construcción de accesibilidad, cobertura universal, participación comunitaria, emancipación y acción intersectorial.

Con el fin de orientar la APS brasileña hacia una APS integral, se aprobó la Política Nacional de Atención Primaria de 2011, que toma como modelo prioritario la Estrategia de Salud Familiar, la cual propone la proximidad territorial, acciones en la comunidad, intersectorialidad, interdisciplinariedad, longitudinalidad, vínculo, corresponsabilidad y articulación entre las acciones de vigilancia y promoción de la salud, y las de prevención de enfermedades $u$ otros problemas de salud y su tratamiento ${ }^{(3)}$.

Para promover transformaciones en el trabajo de los equipos con el propósito de acercarlos a las premisas de la APS integral y la Estrategia de Salud Familiar, se crearon acuerdos y dispositivos que contribuyeran al vínculo entre usuarios y profesionales de la salud, para la co-construcción del cuidado, desde una perspectiva más democrática y participativa ${ }^{(4)}$. Entre estos dispositivos se destacan la clínica ampliada y el apoyo matricial, este último se puede implementar bajo el formato de núcleos de apoyo a la salud familiar, creados en 2008 por el Ministerio de Salud para asistir a los equipos de
Estrategia de Salud Familiar con el apoyo de especialistas centrados en el soporte técnicoasistencial y pedagógico, a fin de favorecer el cuidado integral y la articulación de las redes de atención en salud. El Ministerio de Salud aconseja que todos los núcleos de apoyo a la salud familiar actúen de acuerdo con la metodología de apoyo matricial y estimulen la clínica ampliada.

La clínica ampliada intenta acercar al sujeto a la clínica, incluyendo el cuidado de las dimensiones orgánica/biológica, social y subjetiva. Por su parte, el apoyo matricial, operado por los núcleos de apoyo a la salud familiar, representa una propuesta de trabajo conjunto entre los equipos de especialistas y los de APS, con el objetivo de brindar mayor resolución a través del soporte técnico e intercambio de conocimientos, y menor burocratización en los procesos de referencia y contrareferencia en la red de atención. El apoyo matricial y los núcleos de apoyo a la salud familiar se pueden considerar, por tanto, como facilitadores para efectivizar la clínica ampliada ${ }^{(5,6,7)}$. En cierta medida, vale la pena decir que la clínica ampliada sería una forma de acercamiento a usuarios y profesionales, mientras que el apoyo matricial estaría orientado a reunir a los diversos profesionales de la red; siempre estableciendo relaciones más horizontales y dialógicas, por tanto, más democráticas.

Si bien estos dispositivos son similares o complementan prácticas reconocidas en el escenario internacional ${ }^{(6,8)}$, su implementación ha encontrado importantes obstáculos, que se pueden atribuir, en gran medida, a su carácter antihegemónico de ruptura con el modelo biomédico ${ }^{(5,6,9,10)}$. Por la misma razón, también se reconoce que los profesionales de la salud tienden a formarse en una perspectiva tradicional, de trasmisión del conocimiento, jerarquización y compartimentación del saber ${ }^{(11,12,13,14,15)}$.

Para Mendes-Gonçalves ${ }^{(16)}$, esta forma de operacionalizar las prácticas de salud, que tiende a medicalizar la vida y controlar los cuerpos, tiene su origen en la construcción falaz de que el trabajo de salud puede reducirse a cuestiones técnicas atemporales 
y desconectadas del contexto sociohistórico. En la concepción de Donzelot ${ }^{(17)}$, el modelo hegemónico en salud tiene un alcance amplio e incluye la moralización, normalización y protección de cuerpos y familias desde dos polos: la filantropía-asistencialista y las acciones médico-higienistas.

Si bien Foucault ${ }^{(18)}$ reconoce la posibilidad de producir un cambio en la condición de dominación cuando se establecen relaciones de poder, sostiene que no existe una estrategia preconcebida para la formulación de esta resistencia potencialmente posible. Su construcción dependería, según el autor, de establecer un modelo alternativo para analizar las relaciones de poder, dada la insuficiencia de los modelos existentes, del poder como ley, la prohibición e institución y del poder en términos de relaciones de fuerza (modelo bélico o estratégico).

Ante esta problemática, se formula la propuesta de la formación Paideia ${ }^{(19,20)}$, que parte de referencias constructivistas, dialógicas y de aprendizaje significativo e introduce modificaciones al modelo de los Grupos Balint, con el objetivo de producir efectos en tres dimensiones de manera concomitante: afectos (subjetividad, ser en el mundo), conocimientos (cognición) y poder (en las relaciones institucionales y con los pacientes). La combinación de efectos en estas tres dimensiones busca ampliar la capacidad de análisis e intervención de los profesionales hacia la democratización de los servicios en su sentido amplio: cambiar personas y estructuras ${ }^{(21)}$.

La formación Paideia, haciendo una analogía con el pensamiento de Adorno ${ }^{(13)}$, comprende la formación como una educación que trasciende la información y el fetichismo por la teoría; va más allá de las instituciones educativas y pretende facilitar que los sujetos escapen de su condición de subordinación y conformismo. Se trata de la formación como praxis emancipadora, que favorece el posicionamiento crítico y reflexivo con relación al estado de las cosas.

Dentro de dicha propuesta, la formación Paideia se ha implementado en algunos contextos de capacitación para profesionales de atención primaria dentro de las temáticas de clínica ampliada, gestión compartida, apoyo matricial y apoyo institucional ${ }^{(15,20,22,23,24,25)}$.

Este artículo se inserta en este contexto de implementación de la formación Paideia -tanto para el apoyo matricial, como para profesionales de los equipos de referencia de atención primaria-, el cual deriva de otras dos investigaciones tituladas: "Avaliação do método de Apoio Paideia como estratégia de educação permanente para profissionais do SUS" y "Avaliação de implementação do Método Balint Paideia na formação profissional para a Atenção Primária à Saúde".

Su objetivo es analizar las contribuciones de la formación Paideia a la producción de relaciones de poder basadas en la democracia y la coproducción del cuidado, en el contexto de la APS. Dado que los relatos e iniciativas evaluativas sobre la formación Paideia tienden a privilegiar la presentación de los impactos obtenidos en las dimensiones afectivas y de los saberes ${ }^{(15,20,22,23)}$, resulta necesaria una mayor discusión sobre los aportes de esta propuesta pedagógica en torno a la dimensión del poder. Así, al proponer este análisis, se espera construir condiciones para el debate sobre la democratización de las relaciones en los servicios de salud, especialmente en la APS.

\section{METODOLOGÍA}

El presente estudio se ubica en el campo de la investigación cualitativa ${ }^{(26)}$, en un marco transversal y retrospectivo. Toma como objeto de análisis la dimensión de las relaciones de poder presentes en dos iniciativas pedagógicas de "formación Paideia" Ilevadas a cabo en diferentes momentos: primero, una capacitación en apoyo matricial (2015-2016) que reunió 94 especialistas que realizaban apoyo matricial en APS y segundo, una capacitación en salud familiar (2018-2020), que reunió 149 profesionales, entre ellos 89 pertenecientes a equipos de referencia (responsables de la atención longitudinal en APS) y 60 integrantes de los núcleos de apoyo de salud 
familiar o servicios especializados. En ambas iniciativas se utilizaron las mismas estrategias pedagógicas (discusión de casos, propuestas teóricas y construcción de un proyecto de intervención como trabajo de conclusión del curso). Asimismo, se realizó una evaluación de los procesos de formación mediante diferentes técnicas, con el fin de reunir aspectos cuantitativos y cualitativos del fenómeno, lo que caracteriza la triangulación de métodos, descrita por Minayo ${ }^{(27)}$.

Su seleccionaron como fuentes de datos las encuestas que respondieron los participantes al inicio de la formación Paideia y los diarios de campo producidos durante la observación participante de los investigadores (uno de apoyo matricial y otro de salud familiar), que contenían informes y reflexiones sobre las discusiones y desarrollos de los casos presentados durante las dos iniciativas. Los cuestionarios fueron diseñados para caracterizar tanto el perfil de los participantes, como los elementos de sus prácticas en salud al inicio de la formación.

Dado que la participación en la investigación no era obligatoria, completaron la encuesta $94(100 \%)$ participantes de la formación de apoyo matricial (2015-2016) y 75 (50\%) participantes de la formación en salud familiar (2018-2020). Las encuestas destinadas a ambos grupos compartían preguntas similares referentes a la formación universitaria, al tiempo transcurrido desde la incorporación a la red del SUS y al mismo equipo, como también a datos sociodemográficos, como edad y sexo. Respecto a la formación universitaria, se enumeraron profesiones de acuerdo al Código Ocupacional Brasileño y al Registro Nacional de Establecimientos de Salud del SUS, y cada participante podía elegir solo una opción. Las preguntas relacionadas con la organización del trabajo difieren entre las dos formaciones analizadas, por lo que elegimos solo aquellas más significativas para nuestro artículo (la interrupción del relato del usuario para garantizar objetividad, que se refiere solo a la formación en salud familiar 20182020), y/o aquellas que guardaban similitud interna entre sí: frecuencia de encuentros con los equipos que recibían apoyo o equipos de otros servicios. Las categorías vinculadas a las preguntas sobre organización del trabajo se elaboraron a partir de las directrices de trabajo del Ministerio de Salud destinadas a los núcleos de apoyo a la salud familiar, publicadas en 2009. Para las preguntas referentes a la periodicidad de las acciones, se crearon escala tipo Likert. Al ser variables nominales, se presenta un análisis descriptivo.

A su vez, los diarios de campo tenían como objetivo brindar un panorama de los principales desarrollos de las discusiones, permitiendo vislumbrar los resultados de la formación.

En la investigación etnográfica y en aqueIlas en las que se utiliza la observación participante como técnica de producción de datos, se recomienda que todo se registre en un diario de campo $^{(26)}$. Los diarios remiten a un extracto del discurso institucional "oficial" y constituyen un poderoso dispositivo para la restitución escrita de las implicaciones de los investigadores. Este sería un diario de investigación, similar a un diario íntimo, en el que se relata lo cotidiano de la investigación de campo y las transgresiones a la norma que a veces ocurren ${ }^{(28)}$.

El análisis de los datos obtenidos con los cuestionarios se construyó a partir de la tabulación de las respuestas, seguido del análisis descriptivo de datos, realizado mediante el estudio de las frecuencias absolutas y relativas de las respuestas descompuestas en categorías. Los datos obtenidos a través de los diarios de campo, a su vez, fueron sometidos a al análisis de narrativas, las cuales se insertaron en grillas interpretativas, para luego ser discutidas a la luz de la teoría ${ }^{(29)}$. Finalmente, se realizó la triangulación de datos, reuniendo los diferentes núcleos argumentativos de cada técnica utilizada, tomando como eje rector las principales contribuciones y limitaciones de la formación Paideia para la producción de relaciones de poder basadas en la democracia y la coproducción del cuidado, en el contexto de la APS.

Se cumplieron los requisitos de la Resolución 466/2012 y sus disposiciones complementarias en cuanto a las reglas para la realización de investigaciones con seres humanos, el estudio fue presentado y aprobado 
por el Comité de Ética e Investigación de la Facultad de Ciencias Médicas de la Universidad Estatal de Campinas (UNICAMP), bajo las opiniones 972.660/15 (apoyo matricial) y 2.947.441/18 (salud familiar). Todos los participantes fueron informados sobre los términos de la investigación y aceptaron ser incluidos en el estudio mediante la firma de un formulario de consentimiento informado.

\section{RESULTADOS}

\section{Caracterización de participantes}

En cuanto a la caracterización de los participantes -94 de apoyo matricial y 149 de salud familiar-, en ambas iniciativas, la mayoría eran mujeres $(63 \%)$, de entre 25 y 63 años de edad, procedentes de varias ciudades. En la formación de apoyo matricial (2015-2016), los participantes pertenecían a las ciudades de Campinas (São Paulo), Curitiba (Paraná), Uberlândia (Minas Gerais); mientras que en la formación en salud familiar (2018-2020), a las ciudades de Campinas (São Paulo), Itatiba (São Paulo) y Muzambinho (Minas Gerais). Si bien los integrantes de ambas formaciones participaron de las etapas cuantitativa y cualitativa de estudio, en el marco de la formación en salud familiar, solo completaron la encuesta de evaluación de prácticas profesionales 75 participantes.

En la formación de profesionales de apoyo matricial, identificamos la participación de las siguientes disciplinas: farmacia $(11 \%)$, fisioterapia $(5 \%)$, fonoaudiología $(4 \%)$, medicina $(7 \%)$, nutrición $(4 \%)$, odontología $(3 \%)$, trabajo social $(7 \%)$, terapia ocupacional $(13 \%)$, educación física (4\%), con predominio de psicología (22\%) y enfermería (20\%). La mayoría $(49 \%)$ se había graduado hace menos de cinco años y ya había realizado un posgrado (87\%).

$\mathrm{Al}$ analizar el servicio de origen, se observó que la organización del apoyo matricial a través de los núcleos de apoyo a la salud familiar ocurría de manera heterogénea, evidenciando la complejidad en la organización de las redes de APS, ya que de los 94 participantes, solo 14 de ellos (15\%) formaban parte de los núcleos de apoyo a la salud familiar, $16(17 \%)$ provenían de los servicios del segundo nivel de atención, 15 (16\%) de los centros de atención psicosocial (CAPS) y $10(11 \%)$ de los servicios del tercer nivel de atención. Los 39 participantes restantes (41\%) procedían de otros puntos del sistema, como la gestión y la propia atención primaria, que parecía estar compuesta por un equipo diferente al previsto para la Estrategia de Salud Familiar en el Política Nacional de Atención Primaria $2011^{(3)}$. Cabe recordar que, si bien este es el arreglo preferencial propuesto por el Ministerio de Salud ${ }^{(30)}$ para el apoyo matricial, como metodología de trabajo, también se puede utilizar en otros servicios ${ }^{(19,12)}$.

Sin embargo, cabe señalar que 35 de los profesionales (37\%) informaron actuar con mayor frecuencia en tareas asistenciales que en apoyo matricial y solo 17 de los participantes $(18 \%)$ se dedicaban exclusivamente a realizar este tipo de apoyo. Los demás conciliaban las acciones de apoyo con otras actividades. Es posible entonces observar que el apoyo matricial sigue siendo una actividad poco consolidada dentro de la APS, que compite con la asistencia, que puede considerarse una práctica hegemónica y más habitual en la formación de los profesionales de la salud ${ }^{(12)}$.

Los grupos de capacitación en salud familiar, a su vez, estuvieron integrados por médicos y enfermeras de 48 equipos, alcanzando un 59\% de los participantes de este grupo, y especialistas de 10 equipos de los núcleos de apoyo a la salud familiar, que conformaron el $32 \%$ del grupo, además de 8 profesionales de la gestión $(5,3 \%)$ y 3 en un ambulatorio especializado ( $2 \%)$, cubriendo tres municipios. Entre los especialistas, había un profesor de Educación Física, farmacéuticos, un fisioterapeuta, nutricionistas, psicólogos, terapistas ocupacionales, trabajadores sociales, un oftalmólogo y un pedagogo. Tenían entre 1 y 35 años de recibidos, la mayoría hacía entre 8 y 12 . El $65 \%$ tenía posgrados. El 73,4\% trabajaba solo en el servicio para el que se matriculó en el curso, el $15,2 \%$ en otro servicio, el $8,9 \%$ en otros dos y el $2,5 \%$ trabajaba en más de dos servicios. 
Entre los trabajadores de núcleos de apoyo a la salud familiar, el $45 \%$ informó haber ofrecido actividades de apoyo para cinco o más equipos de salud familiar, el 35\% para tres y el $25 \%$ para dos. A su vez, en la capacitación en apoyo matricial, se observó que el $33 \%$ había trabajado con hasta cinco equipos, el $18 \%$ entre seis y diez equipos, mientras que la mayoría ( $45 \%$ ) manifestó que no consideraban que lo que hacían era apoyo matricial y que, por esta razón, no habían trabajado con ningún equipo.

\section{Caracterización de prácticas sanitarias}

$\mathrm{Al}$ analizar las respuestas de las encuestas sobre la organización del proceso de trabajo, los resultados indican que los profesionales, especialmente los de apoyo matricial, parecen tener dificultades para desarrollar acciones compartidas con los equipos de atención primaria, como se puede apreciar en la Figura 1.

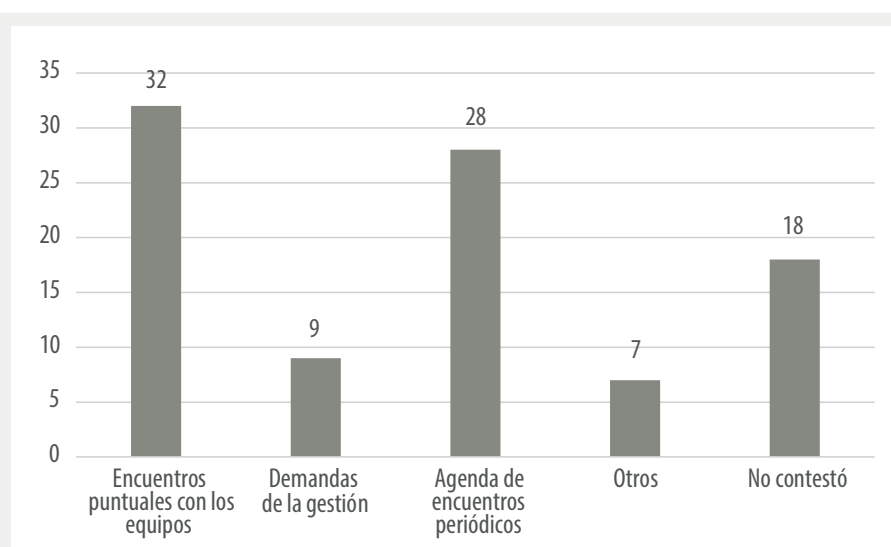

Figura 1. Distribución de la organización de encuentros entre equipos de apoyo matricial y equipos de atención primaria de la salud, según los participantes de la formación de apoyo matricial $(n=94)$. Ciudades de Campinas (São Paulo), Curitiba (Paraná), Uberlândia (Minas Gerais), Brasil, 2015-2016.

Fuente: Elaboración propia.

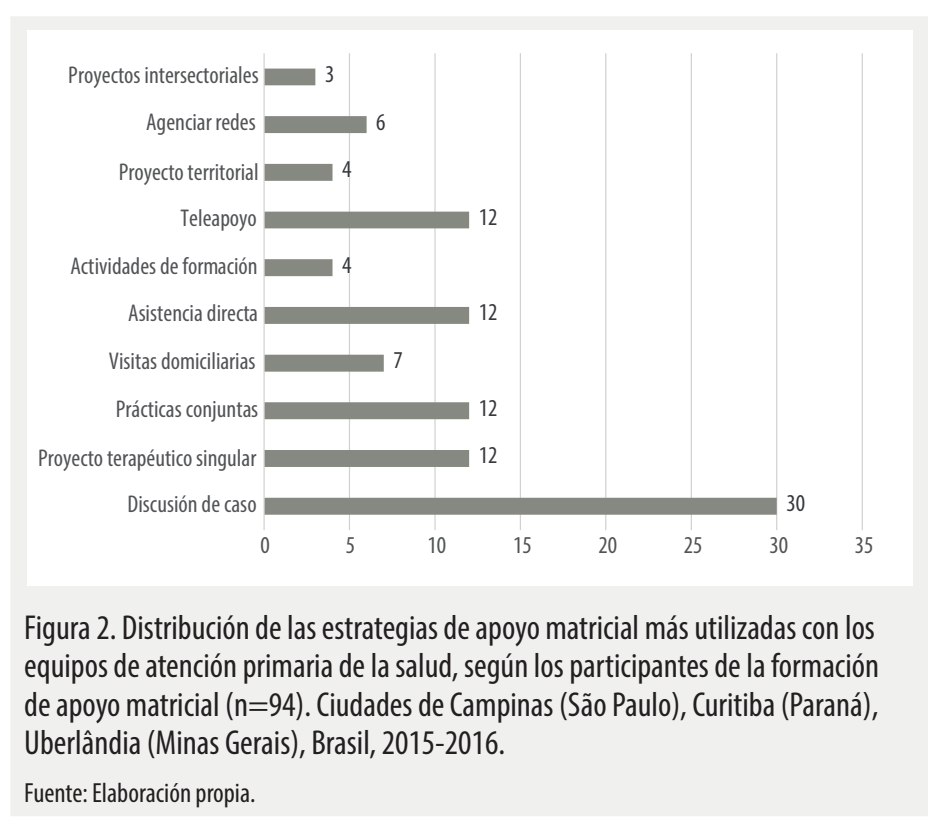

usuarios, aparece con claridad que las acciones conjuntas y la construcción de proyectos terapéuticos singulares presentaron menor frecuencia de respuestas (Figura 2). Incluso los encuentros entre los responsables de brindar apoyo se dan de forma heterogénea y con frecuencia variada (Figura 3), lo que puede no ser suficiente para constituirse como un colectivo organizado para el trabajo ${ }^{(19)}$, con potencial para crear movimientos institucionales orientados al fortalecimiento del apoyo

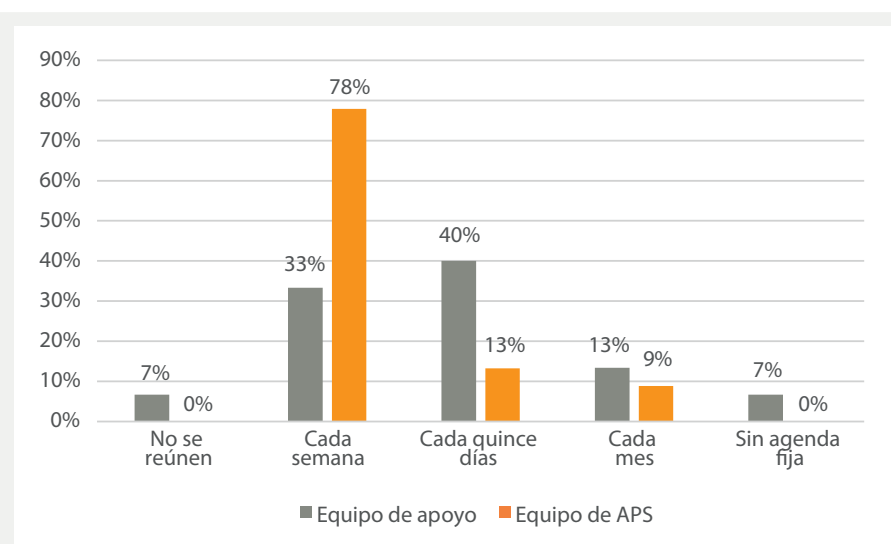

Figura 3. Distribución de la periodicidad en los encuentros de los equipos de apoyo matricial y de los equipos de atención primaria de la salud según los participantes de la formación de salud familiar ( $n=75)$. Ciudades de Campinas (São Paulo), Itatiba (São Paulo) y Muzambinho (Minas Gerais), Brasil, 2018-2020.

Fuente: Elaboración propia. 


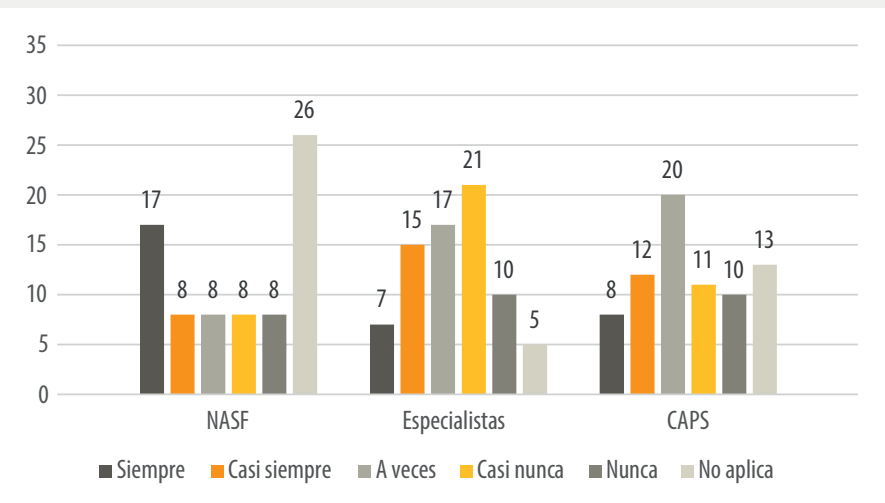

Figura 4. Distribución de la frecuencia de la discusión de casos con otros equipos, según los participantes de la formación de salud familiar $(n=75)$. Ciudades de Campinas (São Paulo), Itatiba (São Paulo) y Muzambinho (Minas Gerais), Brasil, 2018-2020.

Fuente: Elaboración propia.

NASF= Núcleos de apoyo a la salud familiar. CAPS= Centros de atención psicosocial.

matricial. Los equipos de salud familiar, en cambio, suelen reunirse con mayor frecuencia y regularidad (Figura 3 ).

En este sentido, la inconsistencia de las reuniones entre quienes brindan apoyo y los equipos de APS (Figura 1) también parece incompatible con la regularidad semanal de las reuniones del equipo de APS (Figura 3), lo que contribuye a la hipótesis de distanciamiento y escasa implicación entre estas dos modalidades de equipo.

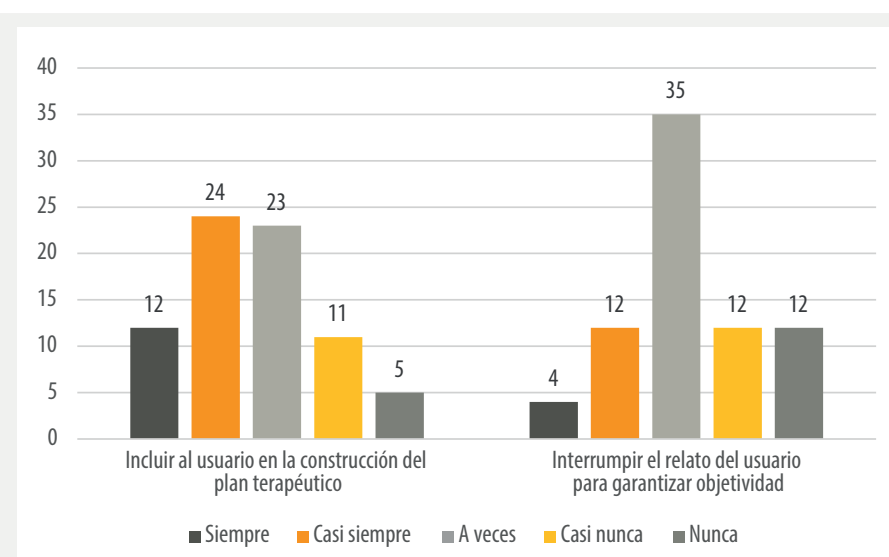

Figura 5. Distribución de la frecuencia de interrumpir el relato del usuario para garantizar objetividad e incluir al usuario en la construcción del plan terapéutico, según los participantes de la formación de salud familiar $(n=75)$. Ciudades de Campinas (São Paulo), Itatiba (São Paulo) y Muzambinho (Minas Gerais), Brasil, 2018-2020.

Fuente: Elaboración propia.
La Figura 4 y la Figura 5 hacen referencia a la relación de los equipos de APS con otros equipos y con los usuarios en la práctica clínica, que aún puede considerarse representativa de la principal actividad de cuidado desempeñada por los profesionales.

El análisis de la Figura 4 señala que, aunque los profesionales de los centros de atención psicosocial se reúnen con frecuencia, solo algunas veces incluyen a profesionales externos o de apoyo en la discusión de casos. Además, según los participantes de la formación de salud familiar, la discusión de casos con otros equipos se da "siempre" con los núcleos de apoyo a la salud familiar con mayor frecuencia, y con los equipos de los centros de atención psicosocial y con especialistas de otros puntos de la red, con menor frecuencia. Estos datos pueden sugerir que la organización de apoyo matricial, especialmente a través del núcleo de apoyo a la salud familiar, aunque necesita avanzar, ha mostrado ser positiva para promover la interacción de la APS con otros saberes, articulando posibilidades para construir la intersectorialidad ${ }^{(10,31)}$.

Sin embargo, para ello es necesario que las discusiones de casos con los equipos de apoyo matricial tengan como objetivo modificar los sistemas de referencia y contrarreferencia, que tradicionalmente son impersonales, burocráticos y con escaso seguimiento del itinerario terapéutico de los usuarios. Asimismo, es importante que estas discusiones puedan diferenciarse de la interconsulta, una práctica muy extendida en los servicios de salud mental y en los servicios hospitalarios, pero que reproduce la fragmentación del cuidado y el gran desequilibrio de poder entre los profesionales. La interacción de los equipos de APS y de apoyo matricial debe estar siempre guiada por los lineamientos de gestión colegiada e interdisciplinariedad, ya que su propuesta es llevar la lógica de la cogestión y del apoyo a las relaciones interprofesionales ${ }^{(19,31)}$.

Según los datos de la Figura 5, el 32\% de los profesionales afirman que casi siempre incluyen a los usuarios en la construcción de su proyecto terapéutico. Si bien la suma de respuestas positivas "siempre" y "casi siempre" prevalece en relación con las demás, cabe 
destacar la alta frecuencia de la alternativa "a veces". También destaca el hecho de que las respuestas "casi siempre" son superiores a "siempre", distinto de lo que se esperaría en un proceso dialógico y coherente con la propuesta de corresponsabilidad del cuidado. Esta participación intermitente de los usuarios dialoga directamente con los datos sobre la interrupción del relato del usuario para garantizar objetividad, que presentó una frecuencia superior a la esperada, considerando el número de respuestas "a veces", "casi siempre" y "siempre".

Estos elementos convergen en el supuesto de la existencia de ciertos rastros de autoritarismo y dominación en la relación que establecen los profesionales en contacto con los usuarios ${ }^{(17,18)}$, desde el inicio de su formación en salud. También apuntan a lo que podría considerarse una escucha selectiva, o participación condicionada, que estaría cerca de lo que Paulo Freire ${ }^{(32)}$ define como el interés parcial que tienen los invasores respecto del pensamiento y el mundo de los invadidos, para poder dominarlos aún más.

\section{Desarrollo de la formación Paideia}

El material de los diarios de campo refuerza esta suposición acerca de la existencia de prácticas autoritarias en los servicios de salud, ya que tiende a retratar las relaciones burocratizadas e interacciones colaborativas incipientes entre los equipos de apoyo matricial y los de APS, y describe una realidad en la acción prescriptiva, rígida y prejuiciosa hacia los usuarios. Para ejemplificar este tipo de acciones. A continuación, presentamos uno de los casos que surgieron en la capacitación en salud familiar.

Mujer de 28 años con diabetes tipo 1 desde los ocho años y sordera congénita, además de problemas de aprendizaje. Vive en una casa cuyo terreno comparte con su suegra y su cuñada. Como ingreso económico recibe asistencia social mediante el "beneficio de prestación continuada". Según el equipo, no realiza actividades de placer u ocio, siendo la encargada de cuidar a su sobrino.

El médico y la enfermera responsables informan el caso de forma confusa, sin una línea de tiempo, sin proyecto de cuidados continuos, lo que denota la falta de práctica en la discusión de proyectos terapéuticos en equipo.

El equipo la conoció hace seis años, cuando fue llevada por su tía, quien exigió que la derivaran a un otorrinolaringólogo para que usara un audífono. Desde entonces, pasó por varias consultas de demanda espontánea, generalmente con quejas de descompensación diabética. El equipo no lograba acercarse a la paciente, pero respondía a las demandas de los familiares. Si bien, dada la sordera, es comprensible que exista dificultad para comunicarse, la unidad contaba con una agente comunitaria de salud que poseía un conocimiento básico en comunicación mediante lengua de señas brasileña, pero el equipo nunca pensó en acercarla más a la paciente.

Comentan que en un momento dado se había conformado un proyecto terapéutico singular por parte de las y los residentes que ingresaron a la unidad, pero aparentemente sin adherencia. En ese momento, el esposo parecía controlar las elecciones de dicho proyecto terapéutico. El abordaje puntual de la paciente se centró en reforzar las posibles complicaciones de la diabetes y tratar de establecer metas, con las que ella dijo tener dificultades, ya que se ponía nerviosa. Luego de que la paciente informara, en una disputa familiar, que había sido abusada sexualmente por el padre, su tía solicitó al equipo que le hagan una "prueba de virginidad", a la que responden positivamente, procediendo a la consulta ginecológica. El informe de abuso, no se abordó con la paciente. En otra ocasión, la familia refiere que la paciente estaría teniendo una relación extramatrimonial, que se discute abiertamente en la unidad, sin su participación. En una tercera situación, la 
paciente dice que desea quedar embarazada, pero el equipo piensa que es un proyecto arriesgado, por la enfermedad de base y la supuesta dificultad de autocuidado, y decide unilateralmente prescribirle anticonceptivos.

Al ser interrogado, el equipo informa que la paciente había obtenido recientemente su licencia de conducir, que mantenía su casa y su apariencia siempre bien cuidadas. Incluso ante estos hechos, el equipo mantenía el discurso de que ella tenía dificultades para el autocuidado, ya que comía muchos dulces, aunque tenía buena comprensión del daño que le podían ocasionar por su enfermedad. (Diario de campo)

Este caso parece ilustrativo de actitudes autoritarias, incluso poco éticas, de los profesionales de la salud, justificadas con base en la técnica. En este caso, el enfoque en la enfermedad de la usuaria y el supuesto deseo de prevenir resultados negativos se utilizaron como motivos para recetar anticonceptivos en contra de su deseo y de diagnosticar un supuesto déficit neurológico. El equipo de salud asume aquí el rol de tutela de los cuerpos en nombre de la moralización y normatividad que pretende la familia(17).

Sin embargo, cabe señalar que, durante la presentación del caso en la capacitación, se evidenció un malestar en los demás participantes, quienes percibieron las intervenciones del equipo, en sus palabras, como violentas: desde el pobre intento para establecer comunicación, la prueba de virginidad, el anticonceptivo recetado porque quería quedar embarazada y el equipo de salud hablando todo el tiempo del riesgo de muerte de la paciente. Algunos participantes, cuestionaron la actitud del equipo de salud, incluso Ilegaron a sugerir un abordaje centrado en el sujeto, abriendo la puerta a las demandas de la usuaria y a la construcción de un proyecto emancipador junto a ella. Aparece allí una brecha para la ruptura de la opresión y, por tanto, para el cuidado dirigido a coproducir la autonomía de los usuarios ${ }^{(33)}$.
En las observaciones realizadas durante la capacitación en apoyo matricial, los profesionales a menudo se quejaban de trabajar de manera aislada, sin compartir los cuidados, sintiendo que la invitación a discutir significaba, muchas veces, una forma diferente de llevar a cabo la vieja práctica de derivar casos.

Los equipos señalan que aún siguen bastante la lógica de la derivación, sin discusión y sin corresponsabilidad de los casos. (Diario de campo)

Uno de los casos más representativos de los impasses vividos en el trabajo de los equipos de apoyo matricial trae como elementos principales la fragmentación del cuidado, la poca interacción entre los equipos y la postura autoritaria que emana de la gestión, para ser reproducida por quienes están a cargo del apoyo, en contacto con usuarios y profesionales de APS.

En respuesta a una solicitud de la dirección central del municipio, el apoyo matricial de fisioterapia debió organizarse por separado del resto de categorías profesionales y tener como objetivo la revisión de listas de espera para atención ortopédica por quejas de dolor musculo-esquelético en la columna, sin causa aparente. Las fisioterapeutas informaron haber encontrado una lista con más de 100 nombres, en solo una de las unidades apoyadas: "Allá había gente que cuando llamamos, ya ni siquiera sentía el dolor. Había personas que ya tenían otra queja e incluso personas que habían fallecido".

La tarea de quienes brindaban apoyo sería volver a convocar a los usuarios en espera y realizar consultas individuales para confirmar la sospecha y la necesidad de derivación. Pero ellas no se sentían cómodas con esto: "No somos ortopedistas y además pareciera que estamos cuestionando la capacidad de los profesionales de la APS que realizaron la derivación". 
Sin embargo, nada de esto se puso en discusión, ni con la dirección local, ni con el equipo apoyado. Las fisioterapeutas iniciaron las consultas individuales y empezaron a sentirse frustradas y desorientadas, sin saber qué hacer y sin la perspectiva de poder completar la tarea. En la presentación a los demás participantes de la capacitación, se les llevó a considerar un nuevo abordaje, en el que la consulta de fisioterapia tendría como finalidad constituir un espacio de escucha y acogida de los pacientes y no una revisión y "limpieza de la lista de espera". También se les animó a cuestionar a la dirección, impugnando sus órdenes mediante la divulgación de esta conducta en una reunión técnica. Y que, si no estaban de acuerdo, tenían derecho a ejercer su autonomía en relación con los pacientes. Al volver a hablar del caso, las fisioterapeutas estaban claramente en otra posición: "Lo que hicimos fue una revolución. Nunca pensamos que podríamos transformar esta tarea encomendada por la dirección en algo placentero para nosotras. Escuchar de un paciente que logró interpretar el dolor como síntoma de un problema emocional y que esto ha transformado su vida es muy gratificante. Incluso si afecta solo a unas pocas personas, vale la pena. Necesitamos ver la clínica como uno de los principales espacios donde debemos reconocer y fomentar el poder de los usuarios. Los pacientes necesitan participar en la construcción de su propio cuidado y tenemos que acercarnos a ellos". (Diario de campo)

En este informe se puede apreciar que las acciones realizadas no llegaron a constituir un enfrentamiento en relación con el cuidado fragmentado, no pretendieron romper la lógica ambulatoria centrada en el cuidado individual, que orientaba el rol de los especialistas, ni tampoco deshicieron las relaciones burocráticas entre profesionales del apoyo matricial y los equipos destinatarios del apoyo.

Como estrategia para lidiar con la frustración que genera este contexto, las personas que brindaban apoyo realizaron una revisión de su práctica clínica y su relación con los usuarios. Fue en la clínica ampliada ${ }^{(5)}$ donde encontraron sentido a su trabajo, acercándolo a la condición de "obra" mencionada por Campos ${ }^{(9)}$, en tanto producto de la realización personal del trabajador. No se puede decir que brindaron apoyo y que establecieron nuevos parámetros, la relación con el equipo de APS, pero es posible que hayan iniciado una micro-revolución en la forma de pensar el cuidado en salud, abriendo el camino para, poco a poco, aprovechar las brechas y así impulsar un movimiento de resistencia, como sugieren Dobies y L'Abbate ${ }^{(34)}$, que defina nuevas direcciones para la APS y el apoyo matricial.

Para algunos de los participantes en ambas iniciativas formativas, la posibilidad de cuestionar su propia práctica y lo que hasta ese momento consideraban como establecido, como ley, puede representar un cambio interno y constituir una posibilidad de construir resistencias ${ }^{(18)}$ y cambiar relaciones de poder, partiendo de modificaciones subjetivas. En una compilación de los extractos del discurso registrados en el diario de campo, se afirma:

...esa lógica de la verticalidad no es algo hablado abiertamente, es algo velado, oculto, tácito. Todavía estamos lejos de donde queremos llegar, pero continuamos en camino. Haber cambiado internamente ya es un primer paso, cambiar el contexto más amplio debe considerarse a mediano y largo plazo. No es posible hacerlo ahora. (Diario de campo)

De esta manera, la propuesta de la formación Paideia, de incrementar la capacidad de análisis e intervención y de operar en las dimensiones de conocimiento, afecto y poder, sigue siendo relevante. Sin embargo, el extracto presentado anteriormente sugiere que los participantes tienden a considerar que estos cambios no ocurren al mismo tiempo, así como que no ocurren en la misma proporción. La expansión de la capacidad de análisis parece preceder a la de intervención, y los impactos sobre la dimensión del afecto, 
asociados a la del conocimiento, aparentemente conducen a la formulación de cambios en la dimensión del poder. La formación, por sí sola, no pudo revertir la relación autoritaria establecida entre profesionales y usuarios.

Sin embargo, no se puede negar el importante aporte de la formación Paideia, como lo indica el presente estudio, en hacer explícitas las relaciones de poder a través de la problematización de las prácticas y la realidad de los equipos. La formación, en cierta medida, pone en análisis la cultura opresora y autoritaria que se reproduce en el conservadurismo institucional, enmascarado como objetividad técnica ${ }^{(16)}$.

\section{CONCLUSIÓN}

El análisis de los resultados obtenidos de las iniciativas de capacitación en apoyo matricial y en salud familiar muestran que aún no se ha podido efectivizar concretamente la propuesta de trabajo contenida en los supuestos de la APS integral, que espera que el paciente participe activamente en la construcción de su proyecto terapéutico, y que su contexto social y aspectos subjetivos siempre sean considerados en el proceso de cuidado. Sin embargo, quedó claro que la formación Paideia es capaz de movilizar a los profesionales para la incorporación de la clínica ampliada y del apoyo matricial en APS y que este movimiento permite compartir el cuidado y la co-construcción de la autonomía junto con los usuarios.

La formación también contribuyó a la percepción de relaciones autoritarias que se establecen en los servicios de salud en diferentes direcciones, con pares, con otros profesionales de la red y con los usuarios. Aún no se ha logrado la deseable democratización para la implementación de la APS integral y, al parecer, será necesario ganar fuerza y cubrir otros campos más allá de la formación, para enfrentar la cultura opresora y prejuiciosa que ha revelado este proceso pedagógico.

Se puede decir que la toma de conciencia acerca de dicho contexto puede iniciarse y ampliarse a través de la formación Paideia, gracias a su propuesta de analizar las dimensiones del afecto y el poder, junto con la producción de conocimiento. Es, por tanto, un impulso de cambio, que debe complementarse con la organización de otros movimientos.

\section{AGRADECIMIENTOS}

Este estudio solo fue posible gracias a la alianza establecida con los municipios participantes y la generosidad de los profesionales de la salud para reflexionar sobre su propia práctica. De la misma forma, expresamos nuestro agradecimiento a los compañeros que forman parte del Colectivo de Estudios y Apoyo Paideia y a la Escuela de Extensión de la Universidade Estadual de Campinas que hicieron posible la realización de los cursos en apoyo matricial y en salud familiar.

\section{REFERENCIAS BIBLIOGRÁFICAS}

1. Giovanella L, Mendonça MHM, Buss PM, Fleury S, Gadelha CAG, Galvão LAC, Santos RF. De Alma Ata a Astana. Atenção Primária à Saúde e sistemas universais de saúde: um compromisso indissociável e direito humano fundamental.
Cadernos de Saúde Pública. 2019;35(3):e00012219. doi: 10.1590/0102-311x00012219.

2. Giovanella L, Mendonça MHM. Atenção Primária à Saúde. En: Giovanella L, Escorel S, Lobato LVC, Noronha JC, Carvalho Al, (orgs.). Políticas e sistemas e saúde no Brasil. 2a ed. Rio de Janeiro: Editora Fiocruz, Cebes; 2012. p. 493-547.

3. Brasil, Ministério da Saúde, Secretaria de Atenção à Saúde, Departamento de Atenção Básica. Política Nacional de Atenção Básica. Brasília: Ministério da Saúde; 2012. (Série E. Legislação em Saúde).

4. Brasil, Ministério da Saúde, Secretaria de Atenção à Saúde. Política Nacional de Humanização da Atenção e Gestão do SUS: Clínica ampliada e compartilhada. Brasília: Ministério da Saúde; 2009. 
5. Campos GWS. Saúde Paideia. 2a ed. São Paulo: Hucitec, 2013.

6. Oliveira MM, Campos GWS. Apoios matricial e institucional: analisando suas construções. Ciência \& Saúde Coletiva. 2015;20(1):229-238. doi: 10.1590/141381232014201.21152013.

7. Campos GWS, Figueiredo MD, Júnior NP, Castro CP. A aplicação da metodologia Paideia no apoio institucional, no apoio matricial e na clínica ampliada. Interface - Comunicação, Saúde, Educação. 2014;18(Supl. 1):983-995. doi: 10.1590/1807-57622013.0324.

8. Starfield B. Atenção primária: equilíbrio entre necessidades de saúde, serviços e tecnologia. Brasília: Unesco, Ministério da Saúde; 2002.

9. Campos GWS. Um método de análise e co-gestão de coletivos: A constituição do sujeito, a produção de valor de uso e a democracia em instituições: O Método da Roda. São Paulo: HUCITEC; 2000.

10. Campos GWS, Domitti AC. Apoio Matricial e equipe de referência: uma metodologia para gestão do trabalho interdisciplinar em saúde. Cadernos de Saúde Pública. 2007;23(2):399-407. doi: 10.1590/S0102$311 \times 2007000200016$.

11. Oliveira GN. Apoio matricial como tecnologia de gestão e articulação em rede. En: Campos GWS, Guerrero AVP, (orgs). Manual de práticas de atenção básica: saúde ampliada e compartilhada. 2a ed. São Paulo: Hucitec; 2010. p. 273-282.

12. Oliveira MM. Apoio matricial na atenção básica de campinas: formação e prática. [Disertación de Maestría]. Campinas: Universidade Estadual de Campinas; 2014.

13. Adorno T. Educação e emancipação: a auto-reflexão da crítica da formação convertida em semiformação. En Adorno T. Educação e emancipação. Rio de Janeiro: Paz e Terra, 1995. p. 117-149.

14. Almeida Filho N. Reconhecer Flexner: inquérito sobre produção de mitos na educação médica no Brasil contemporâneo. Cadernos de Saúde Pública. 2010;26(12):2234 2249. doi: 10.1590/S0102-311X2010001200003.

15. Figueiredo MD. A construção de práticas ampliadas e compartilhadas em saúde: Apoio Paideia e formação. [Tesis de Doctorado]. Campinas: DMPS/FCM/Unicamp; 2012.

16. Mendes-Gonçalves RB. Práticas de saúde e tecnologia: contribuição para a reflexão teórica. En: Ayres RCM, Santos L. Saúde, sociedade e história. São Paulo: Hucitec; 2017. p. 192-250.

17. Donzelot J. A polícia das famílias. Rio de Janeiro: Edições Graal; 1980.

18. Foucault M. Microfísica do Poder. Rio de Janeiro: Paz e Terra; 2015

19. Campos GWS, Cunha GT, Figueiredo MD. Práxis e formação Paideia: apoio e cogestão em saúde. São Paulo: Hucitec; 2013.
20. Oliveira Viana MM, Campos GWS. Formação Paideia para profissionais do Apoio matricial: uma estratégia pedagógica centrada na reflexão sobre a prática. Cadernos de Saúde Pública. 2018;34(8):e00123617. doi: 10.1590/0102-311X00123617.

21. Campos GWS. O anti-Taylor: sobre a invenção de um método para co-governar instituições de saúde produzindo liberdade e compromisso. Cadernos de Saúde Pública. 1998;14(4):863-870. doi: 10.1590/S0102311X1998000400029.

22. Castro CP, Campos GWS. Apoio institucional Paideia como estratégia para educação permanente em saúde. Trabalho, Educação e Saúde. 2014;12(1):29-50. doi: 10.1590/S1981-77462014000100003.

23. Cunha GT. Grupos Balint-Paideia: uma contribuição para a co-gestão e a clínica ampliada na Atenção Básica [Tesis de Doctorado]. Campinas: DMPS/FCM/Unicamp; 2009.

24. Oliveira MM, Pompei DS, Santos JA. Contexto político e apoio: possibilidades de coprodução, experiências Campinas, Curitiba e Uberlândia. En: Campos GWS, Figueiredo MD, Oliveira MM. O apoio Paideia e suas rodas. São Paulo: Hucitec; 2017. p. 301-338.

25. Bedrikow R, Cesar CCF, Duarte CC. Mergulhando e emergindo no Apoio Paideia: reflexões a partir da experiência de formação com um grupo de apoiadores do Ministério da Saúde. En: Campos GWS, Figueiredo MD, Oliveira MM. O apoio Paideia e suas rodas. São Paulo: Hucitec; 2017. p. 241-253.

26. Minayo MCS. O desafio do conhecimento - Pesquisa Qualitativa em Saúde. 11a ed. São Paulo: Hucitec; 2010.

27. Minayo, MCS, Assis, SG, Souza, ER. Avaliação por triangulação de métodos: abordagem de Programas Sociais. Rio de Janeiro: Editora Fiocruz; 2005.

28. Rodrigues HBC. Aprensentação. En: René Lourau na Uerj: Análise Institucional e Práticas de Pesquisa. Rio de Janeiro: UERJ; 1993.

29. Onocko Campos R. Pesquisa qualitativa em políticas, planejamento e gestão em saúde coletiva. En: Barros NF, Cecatti JG, Turato ER, (org). Pesquisa qualitativa em saúde - múltiplos olhares. Campinas: UNICAMP; 2005. p. 261-271.

30. Brasil, Ministério da Saúde. Portaria GM/MS No. 154: Cria os Núcleos de Apoio à Saúde da Família núcleos de apoyo a la salud familiar [Internet]. 2008 [citado 10 oct 2020]. Disponible en: https://tinyurl.com/ wazepehc.

31. Bonfim IG, Bastos ENE, Góis CWL, Tófoli LF. Apoio Matricial em saúde mental na atenção primária à saúde: uma análise da produção científica e documental. Interface: Comunicação, Saúde, Educação. 2013;17(45):287-300. doi: 10.1590/S1414 32832013005000012 .

32. Freire P. Pedagogia do oprimido. 70a ed. São Paulo: Paz e Terra; 2019. 
33. Onocko Campos RT, Campos GWS. Co-construção de autonomia: o sujeito em questão. En: Campos GWS, Minayo MCS, Akerman M, Drumond Júnior M, Carvalho YM, (orgs.). Tratado de saúde coletiva. 2 ed. São Paulo: Hucitec, 2012, p. 669-688.
34. Dobies DV, L'Abbate S. A resistência como analisador da saúde mental em Campinas (SP): contribuições da Análise Institucional. Saúde Debate, 2016;40(110):120133. doi: 10.1590/0103-1104201611009.

\section{FORMA DE CITAR}

Viana MMO, Terra LSV. Formación Paideia en atención primaria: análisis de la democracia institucional y las relaciones de poder en las prácticas laborales en salud. Salud Colectiva. 2021;17:e3298. doi: 10.18294/sc.2021.3298.

Recibido: 23 nov 2020 | Versión final: 7 ene 2021 | Aprobado: 19 ene 2021 | Publicado en línea: 26 may 2021

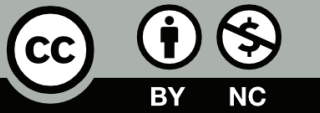

Esta obra está bajo una licencia de Creative Commons Reconocimiento-NoComercial 4.0 Internacional. Reconocimiento - Permite copiar, distribuir y comunicar públicamente la obra. A cambio, se debe reconocer y citar al autor original. No Comercial - Esta obra no puede ser utilizada con finalidades comerciales, a menos que se obtenga el permiso. 